\title{
Improvement of CVRP and MTVRP Solution using Local Search Method and its Implementation Using Google Map
}

\author{
$1^{\text {st }}$ Sapti Wahyuningsih \\ Mathematics Department \\ State University of Malang \\ Malang, Indonesia \\ sapti.wahyuningsih.fmipa@um.ac.id
}

\author{
$2^{\text {nd }}$ Darmawan Satyananda \\ Mathematics Department \\ State University of Malang \\ Malang, Indonesia \\ darmawan.satyananda.fmipa@um.ac.id
}

\begin{abstract}
Vehicle Routing Problem (VRP) is an issue for designing the route for distributing goods from one depot to a number of customers, in such way to minimize cost, every customer visited just once, all routes begin and ends at the depot, as well as the total demand of all customers on the route should not violate capacity of vehicles. There are several variants of VRP; two of them are Capacitated Vehicle Routing Problem (CVRP) and Multiple Trip Vehicle Routing Problem (MTVRP). Routes in such variants built by a certain algorithm, such as Sequential Insertion, Clarke-Wright algorithm, or built randomly as well. These must be refined because they are not provided the best result. This article discusses about refining solution of CVRP and MTVRP. The improvement involves local search method. This method initializes neighborhood list containing six inter-route moves: Shift $(1,0)$, Swap $(1,1)$, Shift $(2,0)$, Swap $(2,1)$, Swap $(2,2)$ and Cross operation. At the main iteration, one of the neighborhoods is randomly selected, and the best move is selected as a new solution. If the new solution is better than the initial solution, four intra-route structures are operated, that is Or-opt, 2-opt, Exchange, and Reinsertion. The algorithm is implemented in a web-based application. Google Map is used to give real data about customer position and the route built, as well as its visualization in the map. From the test, the algorithm can improve the initial result built by Sequential Insertion and Clarke-Wright algorithm, in the form of shorter route and number of vehicles used.
\end{abstract}

Keywords-local search, CVRP, MTVRP, google map

\section{INTRODUCTION}

Distribution system is the process of distributing products from the depot to the customer and is an important factor in determining the mileage or cost. Vehicle Routing Problem (VRP) is one of the problems that can be used to design the distribution route from the depot to a number of customers whose locations are scattered, with minimal cost. The route is designed so that each customer is visited exactly once with one vehicle, all routes start from and end at the depot. In addition, the total demand from all customers on a single route should not violate vehicle capacity. Capacitated Vehicle Routing Problem (CVRP) is one variant of VRP where a number of customers are served by a number of equal-capacity vehicles. In other distribution problems, the vehicle performs several travel routes by returning to the depot as customer demand exceeds the existing capacity of the vehicle but still meets the operating time limits. This problem is another VRP variant called Multiple Trip Vehicle Routing Problem (MTVRP).

CVRP and MTVRP can be solved with various algorithms. Some examples include using Clarke and Wright Algorithm, Sequential insertion heuristic algorithm, and Genetic Algorithm [1][2][3]. Determination of MTVRP solution that has been studied is with Tabu Search algorithm, and Variable Neighborhood Descent Algorithm [4,5]. The solution obtained by the algorithm is a global solution and based on the literature review, it is not necessarily an optimal solution. There are several methods to improve the solution to obtain the optimal solution, one of them with the improvement of the solution using local search method.

Discussion of local search can be seen on S. Akpinar (2016) by using Hybrid Large Neighborhood Search method for CVRP, and Variable Neighborhood Search algorithm by M. Cheikh et al. (2015) Local search discussion is also implemented on other VRP variants i.e. VRP with Time Window, Heterogeneous Fleet, and Multiple Trip VRP with Backhauls [6-10].

Determination of initial solution to complete CVRP and MTVRP is performed by using sequential insertion algorithm and Clarke-Wright algorithm, fixing solution with local search method and its implementation using google map that will be reviewed in this article.

\section{Formulation of CVRP and MTVRP}

CVRP mathematical formulation to minimize the total cost of all vehicles are as follows:

- The objective function is to minimize cost

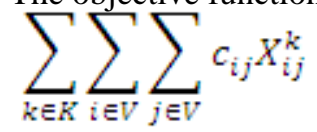

$\mathcal{X}_{i j}^{k}= \begin{cases}1 & \text { jika kendaraan } k \text { dijalankan dari titik } i \text { ke titik } j \text { dengan } i \neq j \\ 0 & \text { jika kendaaraan } k \text { tidak dijalankan dari titik } i \text { ke titik } j\end{cases}$

- $\quad$ Every customer is visited exactly once

$$
\begin{array}{ll}
\sum_{k \in K} \sum_{i \in V} X_{i j}^{k}=1_{j} & \forall j \in V \backslash\{0\} \\
\sum_{k \in K} \sum_{j \in V} X_{i j}^{k}=1_{j} & \forall i \in V \backslash\{0\}
\end{array}
$$


- The total demand of each customer in one route should not exceed the capacity of the vehicle

$$
\sum_{j \in V} d_{j}\left(\sum_{i \in V} X_{i j}^{k}\right) \leq Q_{s} \quad \forall k \in K
$$

Notes:

- $\quad V$ : depot and customer

- $\quad k$ : vehicles used

- $d_{j}$ : demand of customer $j$ with $j \in\{1,2, \ldots, n\}$

- $Q$ : capacity of vehicle $k$

MTVRP mathematical formulation to minimize the total cost of all vehicles are as follows:

- The objective function:

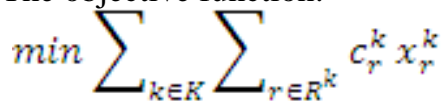

- To ensure that the total time of distribution of a vehicle does not exceed the maximum given time:

$$
\sum_{r \in R^{k}} T_{r}^{k} x_{r}^{k} \leq T_{\text {MAX }} \forall k \in K
$$

- To ensure that each vehicle passes at least 1 route $\sum x_{r}^{k} \geq 1 \forall k \in K$

Notes:

- $K$ : set of vehicles in depot, with $K=\{1,2, \ldots, m\}$

- $R^{k}$ : set of routes passed by vehicle $k$, with $R=\left\{R^{1}, R^{2}, \ldots, R^{k}\right\}$

- $\quad r$ : route index

- $c_{r}^{k}$ : vehicle mileage when traveling route $\mathrm{r}$

- $T_{r}^{k}$ : time needed by vehicle $k$ to travel route $r$

- $T_{M A X}$ : maximum travel time for a vehicle

- $\quad x_{r}^{k}$ : binary integer; valued 1 if vehicle $k$ passes route $r$, and valued 0 for other condition

\section{METHODS} are:

Steps taken in finding CVRP and MTVRP in this research

1. The literature study of algorithms in finding solutions of CVRP and MTVRP, as well as algorithms of local search

2. Creating a web-based application to solve CVRP and MTVRP problem that implements local search

3. Implement the application using standard data or field data

4. Analyze the algorithm and result of application to find the effectiveness

Determination of the initial solution for completing CVRP and MTVRP in this article is done by using Sequential Insertion and Clarke-Wright algorithm. The initial solution uses the Sequential Insertion algorithm as follows:

1. Choosing an unassigned vehicle

2. Choosing the first customer (seed customer), that is the customer who has the furthest distance from the depot.
3. Adding customers to a new route; the customer selected is the on that has the closest distance to the first customer. Repeat until you no more customer to insert, go to 4.

4. Move the route to a smaller vehicle. If it has been moved back to 3 if it does not return to 1 . If the total driving time still has not reached the operating time, then the vehicle may take a new route again.

The initial solution uses the Sequential Insertion algorithm as follows:

1. Calcuate saving with $S_{i j}=c_{i 0}+c_{0 j}-c_{i j}$ for $i=1, . ., n-1$ and $j=i+1, . ., n$

2. Sort $S_{i j}$ in descending order

3. Build and choose routes from largest to smallest $S_{i j}$.

4. Route expansion: Based on the route that has been formed in step 3, the merged route occurs if it does not violate the specified constraint. The process of extending the route is continued until the number of demand in one route does not exceed the capacity of the vehicle.

5. If all $S_{i j}$ are selected and there exist $i \in V /\{0\}$ not included in the route in step 4 , then a new route will be established.

6. The route extension process is terminated after all is covered in all routes that have been established in steps 4 and 5. Customers selected on the previous route should not be re-elected in the next route selection

\section{RESULTS AND DISCUSSION}

\section{Improvement of CVRP and MTVRP solution}

The solution of CVRP and MTVRP using Sequential Insertion algorithm and Clarke-Wright algorithm can be improved by using local search method. Local search method is an iterative procedure that aims to find the best solution by minimizing the evaluation function. Local search procedures start from forming travel routes that involve the customer, customer demand, and the distance between depot-customer and customer-customer. The routes formed are called initial solutions, then this initial solution is tested for optimization. Optimization is tested by comparing the initial solution evaluation function with the evaluation function of other solutions. Another solution is obtained from the operation of neighborhoods on local search. If the result of the evaluation function of the initial solution is worse than the evaluation function of other solutions, then the other solution becomes the new starting solution. This process is repeated until all solutions are completely compared. If a final solution obtained is better than the original solution, then the new solution is accepted to be an improved solution.

Solution CVRP and MTVRP with local search methods used in this article using inter-route and intra-route improvements. Six inter-route structures are described as follows.

1. Shift $(1,0)$ : One customer $i$ is moved from route $r_{1}$ to $r_{2}$.

2. Swap $(1,1)$ : Interchange between customer $i$ from route $r_{1}$ and customer $j$ from route $r 2$. 
3. Shift $(2,0)$ : Two adjacent customers $i$ and $j$ (or an arc ( $i$, $j$ )) are moved from route $r_{1}$ to route $r_{2}$. The transfer of the opposite $\operatorname{arc}(j, i)$ is also considered.

4. Swap (2,1): Interchange between adjacent customers $i$ and $j$ from route $r_{1}$ and customer $k$ from route $r_{2}$. As in the previous case, the opposite arc $(j, i)$ is also considered.

5. Swap (2,2): Interchange between adjacent customers $i$ and $j$ from route $r_{1}$ and two other adjacent customers $k$ and $l$, from route $r_{2}$. The opposite $\operatorname{arcs}(j, i)$ and $(l, k)$ are also considered, thus yielding 4 possible combinations.

Cross: edges between the connected customer $i$ and $j$ of route 1 and the edges between customer $\mathrm{k}$ and 1 of route 2 is removed. Then, two sides $(\mathrm{i}, \mathrm{l})$ and $(\mathrm{k}, \mathrm{j})$ are inserted.

Meanwhile, four intra-route structures are described as follow:

1. Or-opt: one to three consecutive nodes are moved into other position in the route

2. Two-opt: two un-adjacent edges are removed, and two new edges are formed then to make a new route

3. Exchange: exchanging two nodes position. This is another version of swap $(1,1)$, but it applied to the same route

4. Reinsertion: a node is removed, and inserted into other position in the route

Local search method is to find the initial solution, make improvements, and get the optimum conditions. Solution improvement process using local search method is done repeatedly until the optimum condition is obtained. The optimum condition is achieved when all neighborhood structures are applied and can not be improved any more.

\section{Implementation using google map}

Implementation of algorithms is made in a web-based application with data input required are the position of the point, the demand of each customer, vehicle capacity, and vehicle speed. Google Map is used to provide real data about the distance between points in the form of the distance matrix, and display of the interactive and real map. Furthermore, the program will perform the process of search and repair routes.

The output of the application of the implementation results in the form of text, images, and graphics. Image information is the graph of the route formed. The text output contains descriptive information about the initial solution, new solutions for each iteration and final solution for CVRP and MTVRP issues accompanied by total distance and maximum travel time. While the graph contains information about the acquisition of solutions on each iteration.

Here some pictures related to the program:

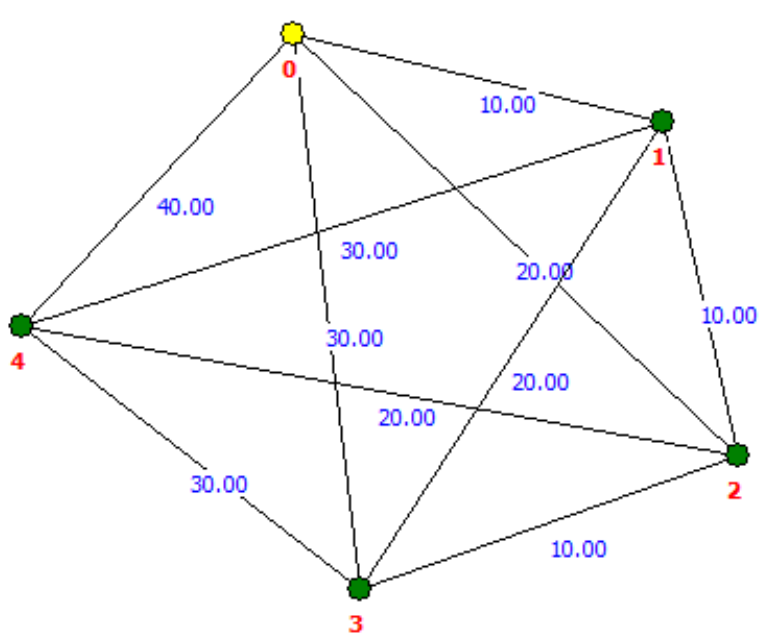

Fig. 1. Graph model

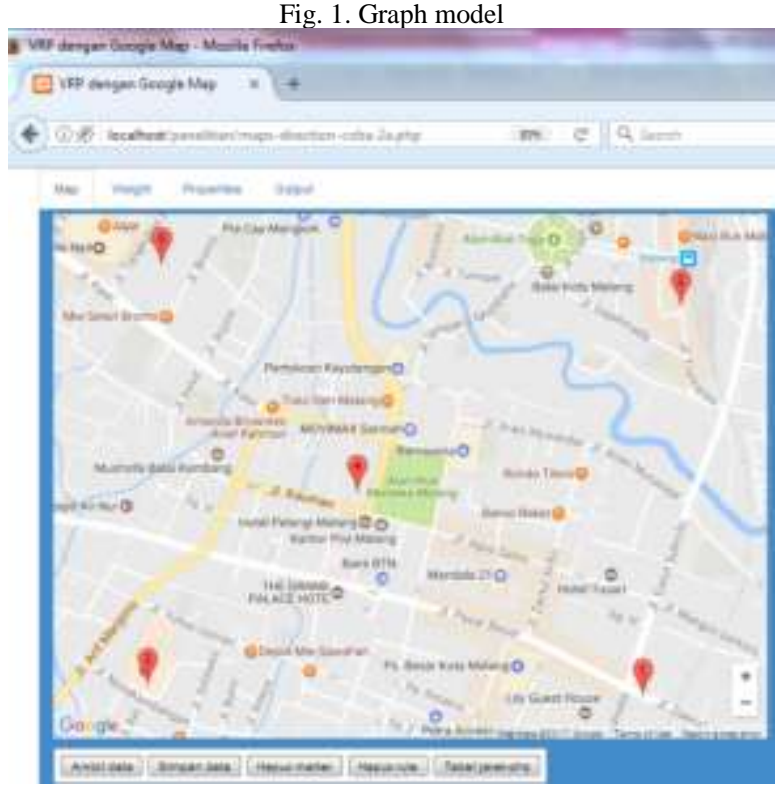

Fig. 2. Markers in map to determine depot and customer location.

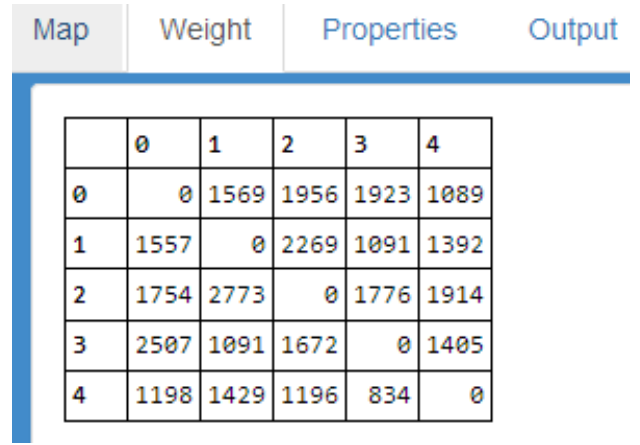

Fig. 3. Real distance from Google's Distance Matrix Service. 


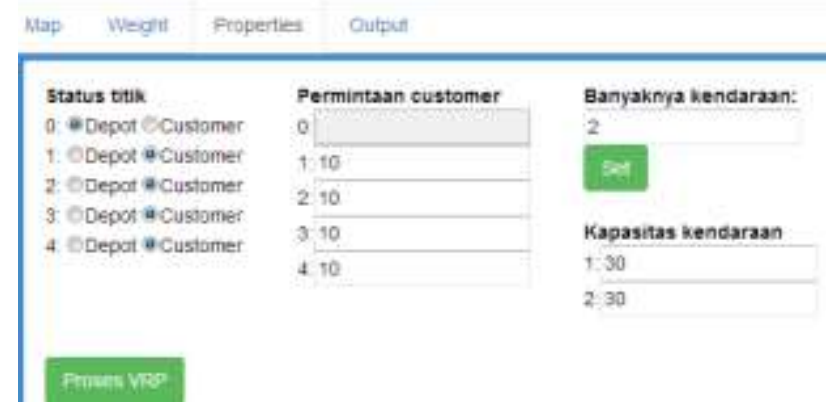

Fig. 4. Input of customer's demand.

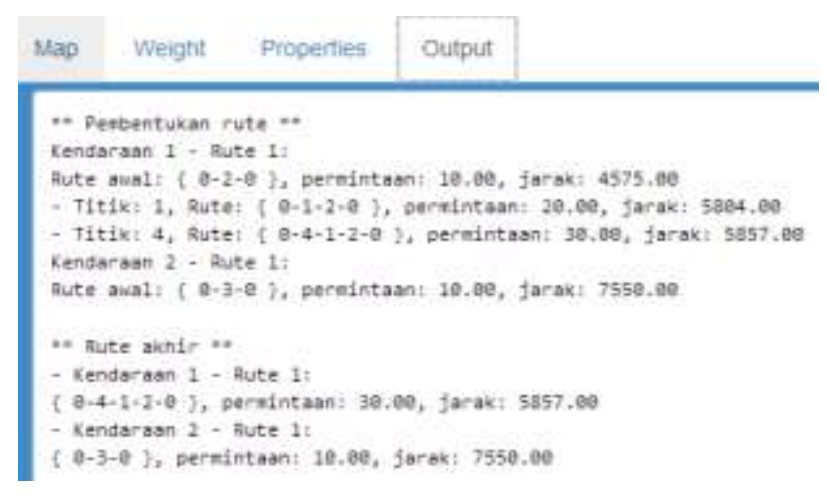

Fig. 5. Output in textual form

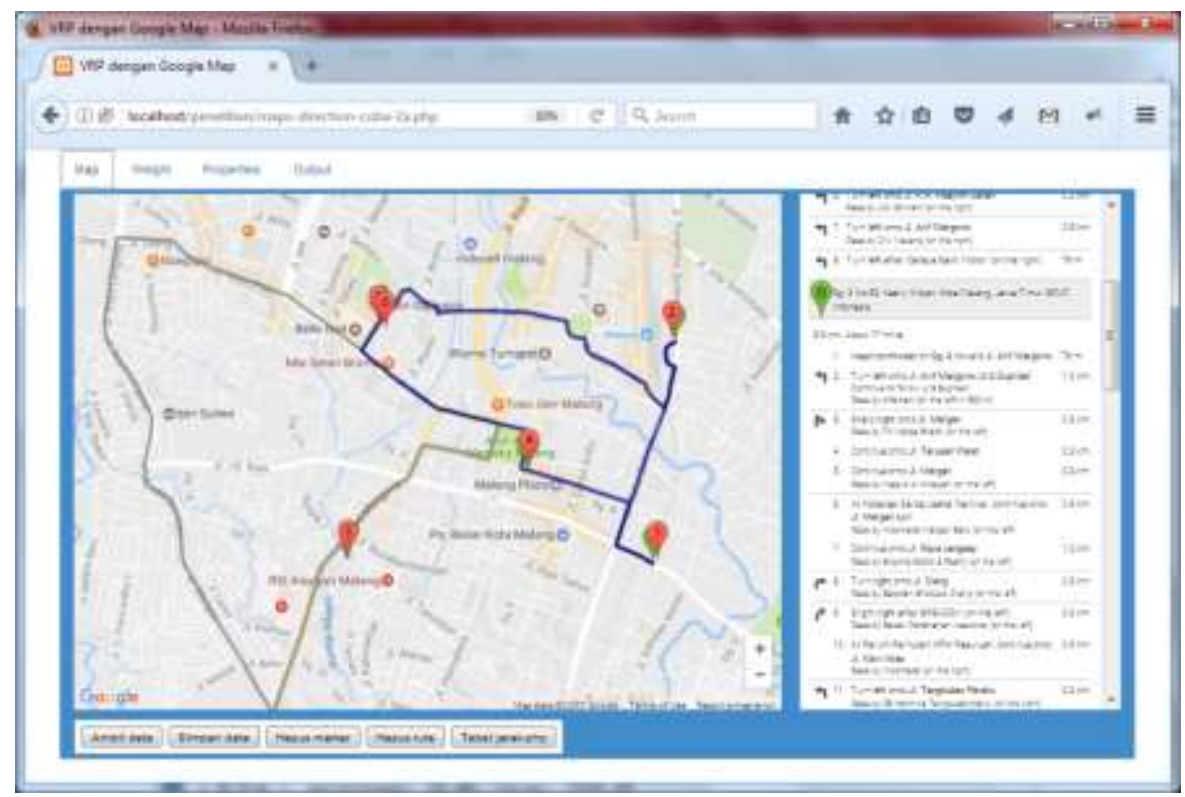

Fig. 6. Route as solution in graphical form in the map

\section{CONCLUSION}

The discussion in this article is about the determination of the initial solution of CVRP and MTVRP using sequential insertion algorithm and Clarke-Wright algorithm, then the initial solution is improved by local search method. Application developed is web-based, by utilizing the Google Map service to display a map of an area and get the distance between places appropriately. For further process improvement with local search this can be applied to other variants of VRP and its combination.

\section{ACKNOWLEDGEMENTS}

This article is part of research entitled "Utilization of Google Map Services for the Application of VRP Problem Solving" (year 2007, the first year of two years planned). The research is funded by Directorate of Research and Community Services (DRPM), Indonesian Ministry of Research, Technology, and Higher Education (Kemenristekdikti).

\section{REFERENCES}

[1] L. Caccetta, M. Alameen, and M. Abdul-Niby, "An improved clarke and wright algorithm to solve the capacitated vehicle routing problem", Engineering, Technology \& Applied Science Research vol. 3, no. 2, pp. 413-415, 2013.

[2] J. W. Joubert, and S. J. Claasen. "A sequential insertion heuristic for the initial solution to a constrained vehicle routing problem", ORiON vol. 22, no. 1, pp. 105-116, 2006.

[3] S. Salhi, and R. J. Petch, "A GA based heuristic for the vehicle routing problem with multiple trips", Journal of Mathematical Modelling and Algorithms, vol. 6, no. 4 pp. 591-613, 2007.

[4] F. Alonso, M. J. Alvarez, and J. E. Beasley, "A tabu search algorithm for the periodic vehicle routing problem with multiple vehicle trips and accessibility restrictions", Journal of the Operational Research Society, vol. 59, no. 7, pp. 963-976, 2008. 
[5] A. Chbichib, R. Mellouli, and H. Chabchoub, "Profitable vehicle routing problem with multiple trips: Modeling and variable neighborhood descent algorithm", American Journal of Operational Research, vol. 2, no. 6, pp. 104119, 2012.

[6] S. Akpinar, "Hybrid large neighbourhood search algorithm for capacitated vehicle routing problem", Expert Systems with Applications, vol. 61, pp. 28-38, 2016.

[7] M. Cheikh, M. Ratli, O. Mkaouar, and B. Jarboui, "A variable neighborhood search algorithm for the vehicle routing problem with multiple trips", Electronic Notes in Discrete Mathematics, vol. 47, pp. 277-284, 2015.

[8] L. En-nahli, S. Afifi, H. Allaoui, and I. Nouaouri, "Local Search Analysis for a Vehicle Routing Problem with Synchronization and Time Windows Constraints in Home Health Care Services", IFAC-PapersOnLine, vol. 49, no. 12, pp. 1210-1215, 2016.
[9] P. H. V. Penna, P. H. Vaz, A. Subramanian, and L. S. Ochi, "An iterated local search heuristic for the heterogeneous fleet vehicle routing problem", Journal of Heuristics, pp. 1-32, 2013.

[10] N. Wassan, N. Wassan, G. Nagy, and S. Salhi, "The Multiple trip vehicle routing problem with backhauls: Formulation and a two-level variable neighbourhood search", Computers \& Operations Research, vol. 78, pp. 454-467, 2017. 